\title{
Besondere Qualitätsaspekte von Tibetischen Arzneimitteln - Erkenntnisse aus über 40 Jahren Erfahrung in der Herstellung in der Schweiz
}

\author{
Alexandra Schwabl Erich Gämperle
}

Padma AG, Hinwil, Schweiz

\section{Schlüsselwörter \\ Tibetische Medizin . Pflanzliche Vielstoffgemische . GMP · Qualität · Herstellung}

\section{Zusammenfassung}

Tibetische Rezepturen sind Komplexformeln aus pflanzlichen und mineralischen Inhaltsstoffen. Die Padma AG stellt seit über 40 Jahren ausgewählte Rezepturen der Tibetischen Medizin in der Schweiz her. Dabei werden moderne Qualitätsnormen und Good Manufacturing Practice (GMP)-Richtlinien befolgt, die die Qualität von den Ausgangsstoffen über die Herstellungsprozesse bis hin zum Fertigprodukt garantieren. Ziel ist es, diese wertvollen Formeln Menschen im Westen in gleichbleibend hoher Qualität "made in Switzerland" zur Verfügung zu stellen. Die Herstellung nach modernen Qualitätsrichtlinien ist anspruchsvoll, ressourcenintensiv und benötigt spezielles Know-how z.B. in der Rohstoffbeschaffung und der Qualitätsanalytik unter Einbezug von pharmakognostischem und botanischem Wissen.

\section{Einleitung}

Die Rezepturen der Tibetischen Medizin gehören zu den wertvollsten Kostbarkeiten des globalen Arzneimittelschatzes. Als pflanzliche Komplexformeln weisen sie ein sehr breites pharmakologisches Spektrum auf. Durch ihre Vielstofflichkeit und die Zusammenstellung nach jahrhundertelanger empirischer Erfahrung [1] bieten Tibetische Rezep-

Hinweis: Padma 28 (Swissmedic No. 58436) ist in der Schweiz auch unter den Namen Padmed Circosan (Swissmedic No. 60131) und Arteria-vita (Swissmedic No. 62863) erhältlich und kassenzulässig.

\author{
Keywords \\ Tibetan Medicine - Herbal multi-compound mixtures . \\ GMP · Quality · Manufacturing
}

\section{Summary}

Special Aspects of Quality of Tibetan Medicines Insights from over 40 Years of Manufacturing Experience in Switzerland

Tibetan recipes are complex formulas from plant and mineral ingredients. Padma Inc. has been producing selected formulas from Tibetan Medicine in Switzerland since more than 40 years. Modern quality standards and Good Manufacturing Practice (GMP) guidelines are followed, ensuring the quality of the raw materials through the manufacturing processes to the finished product. The aim is to provide these valuable formulas to people in the West in a consistently high quality 'made in Switzerland'. The production according to modern quality standards is challenging, draws on many resources, and requires specialized expertise, e.g. in the procurement of raw materials and the quality analysis including pharmacognostic and botanical knowledge.

turen wirksame und in der Regel gut verträgliche Therapieoptionen, dies besonders auch bei chronischen Erkrankungen und solchen mit multikausaler Ätiologie [2, 3]. Der Reichtum an weitgehend pflanzlichen Inhaltsstoffen ist durch den für jede Rezeptur charakteristischen Geruch und Geschmack direkt erfahrbar.

Der folgende Artikel gibt einen kurzen Überblick über die speziellen Herausforderungen bei der Good Manufacturing Practice(GMP)-konformen Herstellung Tibetischer Rezepturen und beleuchtet jene Schlüsselstellen, die aufgrund der außergewöhnlich komplexen Zusammensetzung der Vielstoffgemische der Tibetischen Medizin besonders zu beachten sind.

\begin{tabular}{ll}
\hline KARGER & @ 2013 S. Karger GmbH, Freiburg \\
Fax +497614520714 & 1661-419/13/0208-0014\$38.00/0 \\
$\begin{array}{l}\text { Information@Karger.com } \\
\text { www.karger.com }\end{array}$ & $\begin{array}{l}\text { Accessible online at: } \\
\text { www.karger.com/fok }\end{array}$
\end{tabular}




\section{Herstellung in Europa}

Die Padma AG stellt sich seit über 40 Jahren der Herausforderung, komplexe Rezepturen der Tibetischen Medizin in hoher Qualität herzustellen. Daraus folgte bereits 1969 der strategische Entscheid, die Herstellung pionierhaft in der Schweiz durchzuführen.

Schon zu Beginn war klar, dass das enorme Potenzial dieser Rezepturen für Menschen im Westen nur dann voll ausgeschöpft werden kann, wenn die Produkte nach modernen Qualitätsstandards hergestellt werden. Dabei gelten, wie auch bei der Herstellung konventioneller Arzneimittel, vor allem die Standards der GMP [4]. Tibetische Rezepturen nehmen unter den pflanzlichen Arzneimitteln insofern eine spezielle Rolle ein, als dass sie Vielstoffrezepturen aus mindestens drei, meist aber bis zu Dutzenden von Rohstoffen sind [5]. Mit dieser Vielstofflichkeit stellen sie besonders hohe Anforderungen an alle Bereiche der Arzneimittelherstellung, von der Definition der Qualität über die nachhaltige Beschaffung hochwertiger Rohstoffe bis hin zur analytischen Prüfung im Rahmen der Qualitätskontrolle.

\section{Pflanzliche Ausgangsstoffe}

Für jeden Rohstoff werden in einer Spezifikation die verschiedenen Qualitätsanforderungen zusammengefasst. Darin wird die geforderte Qualität definiert und die zu verwendenden Analyseparameter und -methoden beschrieben. Für Medizinalpflanzen und Pflanzendrogen, die auch im europäischen Raum bekannt sind, werden für die Spezifikationen die Monographien der Europäischen Pharmakopöe herangezogen [6]. Da für die meisten Rohstoffe der Tibetischen Rezepturen (noch) keine offiziellen Monographien existieren, mussten für viele Rohstoffe firmeneigene Monographien bzw. Spezifikationen erstellt werden, was unter Einbezug von pharmakognostischem, botanischem und chemisch-analytischem Know-how erfolgte. Bei der Spezifikation und der Qualitätskontrolle sind insbesondere Identität, Qualität und Reinheit Schlüsselfaktoren.

\section{Identität}

Durch die Identitätsprüfung wird sichergestellt, dass es sich um die richtige botanische Spezies und den zu verwendenden Pflanzenteil handelt. Dies kann bei getrockneten Pflanzendrogen, besonders wenn sie bereits in Pulverform geliefert werden, schwierig sein. Zur Identitätsprüfung werden unter anderem makro- und mikroskopische Merkmale analysiert (Abb. 1) sowie organoleptische Eigenschaften (Geruch und Geschmack) herangezogen. Weiter kommen z.B. Dünnschichtchromatographie-Analysen zur Anwendung (Abb. 2) [7].

\section{Qualität}

Die Qualität wird durch die Mindestkonzentration eines oder mehrerer chemischer Inhaltsstoffe definiert. Im Gegensatz zu chemischen Monopräparaten ist die Festlegung dieser qualitätsbestimmenden Substanzen bei Pflanzendrogen mit ihren vielen chemischen Inhaltsstoffen sehr schwierig. Sie können, müssen aber nicht, pharmakologisch aktive Stoffe sein. Meist werden Markersubstanzen gewählt, die als besonders empfindlich gelten und somit als Indikatoren für die Einhal-

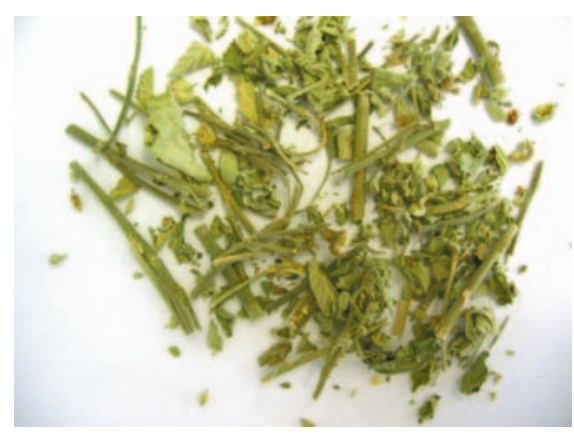

Abb. 1. Sidakraut (Sidae cordifoliae herba): a) Rohdroge und b) Mikroskopie, Vergrößerung 312,5 ×, charakteristisches Sternhaar.

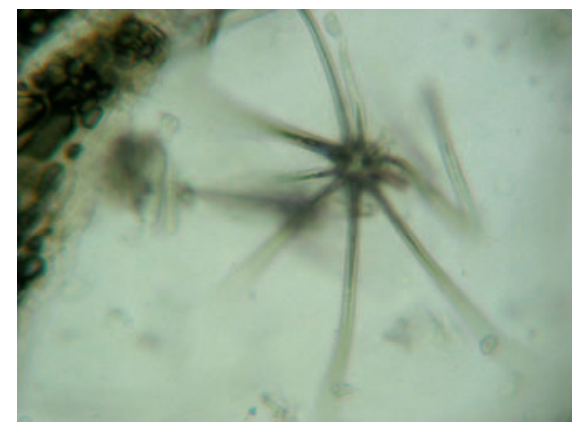

Abb. 2. Vogelknöterichkraut (Polygoni avicularis herba): Dünnschichtchromatographie (Fingerprint) von 4 Proben, fotografiert unter UV-Licht (366 nm). In den beiden äußeren Banden als chemische Marker (von oben nach unten): Kaffeesäure, Hyperosid, Chlorogensäure.

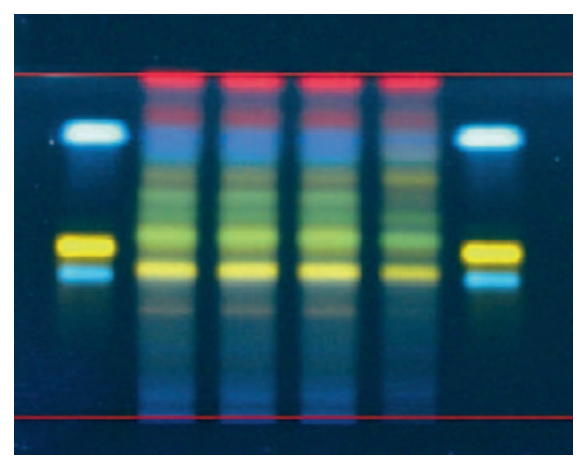

tung des optimalen Erntezeitpunkts, der validierten Verarbeitungsprozesse und der Lagerbedingungen dienen können [8] (Abb. 2 und 3).

\section{Reinheit}

Pflanzliche Ausgangsstoffe werden mit Wasserdampf keimreduzierend behandelt und fein vermahlen. Jeder Ausgangsstoff wird auf die Kontamination z.B. mit Pestiziden, Schwermetallen oder Aflatoxinen überprüft, und die Einhaltung der vorgegebenen Grenzwerte wird sichergestellt. Ebenso sind die Werte für die mikrobiologische Reinheit einzuhalten.

\section{Mögliche Fehlerquellen und Qualitätsrisiken}

Mit einer sorgfältigen Risikoanalyse werden im Vorfeld des Produktionsprozesses mögliche Fehlerquellen und Qualitätsrisiken identifiziert und evaluiert. Folgende Punkte werden beurteilt: a) Art und Ausmaß des potenziellen Fehlers in Bezug auf Wirksamkeit und Sicherheit des Fertigprodukts, b) Auftrittshäufigkeit sowie c) Entdeckungswahrscheinlichkeit. Aufgrund dieser Analyse werden Maßnahmen zur Fehlerprävention beschlossen und umgesetzt.

Allgemein ist in der Phytopharmazie das Risiko für das Auftreten von Fehlern abhängig von der Qualität der Liefe- 
Abb. 3. Bestimmung von Piperin in Padma Digestin mit Hochleistungsflüssigkeitschromatographie (HPLC).

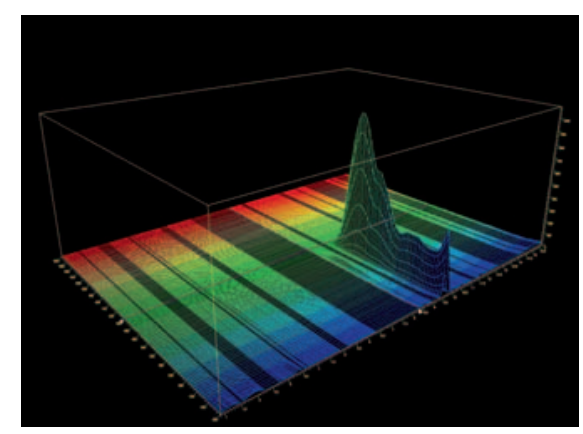

Auch das Fertigprodukt wird mittels Qualitätskontrollen und -analysen auf die Konformität mit seiner Spezifikation überprüft. Die darin definierten Eigenschaften sind so gewählt und von den zuständigen Arzneimittelbehörden genehmigt, dass die Wirksamkeit und Sicherheit des Produkts über den angegebenen Zeitraum garantiert ist. Zusätzlich werden deshalb Haltbarkeitsanalysen bzw. Stabilitätsstudien durchgeführt. Als freigegebenes Endprodukt hat etwa das Produkt Padma ${ }^{\circledR} 28$ mit seinen 22 Rohstoffen insgesamt etwa 300 Einzelprüfungen überstanden, bevor es als Fertigprodukt in den Handel kommt.

\section{Schlussfolgerung}

ranten bzw. landwirtschaftlichen Produzenten. Deren Qualität wird durch die zwingende Befolgung der Richtlinien der Good Agricultural and Collection Practice (GACP) durch alle Padma-Lieferanten gewährleistet [9]. Weiter erleichtert ein direkter Kontakt zu den Produzenten, im besten Fall eine langjährige Zusammenarbeit, die Umsetzung von Qualitätssicherungsmaßnahmen und damit die Fehlerprävention.

Dank dieser Prüfkaskade und der Tatsache, dass sämtliche Rohstoffe vor der Weiterverarbeitung durch das firmeneigene Qualitätskontrolllabor auf Einhaltung der Spezifikation geprüft werden, wird die Bereitstellung hochwertiger Rohstoffe für die Produktion garantiert.

\section{Qualitätskriterien des Fertigprodukts}

Entscheidend für die Qualität des Fertigprodukts ist die Qualität der Ausgangsstoffe sowie der Herstellungsprozesse, wobei natürlich auch die Packmittel wie z.B. Blisterfolien und Hilfsstoffe wie Kapselhüllen den in eigenen Spezifikationen definierten Qualitätsansprüchen genügen müssen.

Indem die Padma AG vor einigen Jahren weitgehend von Tabletten auf die Kapselform umgestellt hat, konnte einerseits die Zahl der Hilfsstoffe weiter reduziert werden, andererseits stellt das lose Pflanzenpulver in den Kapseln die ursprüngliche galenische Form der Tibetischen Rezepturen dar. Dies ist also ein Schritt näher zum Ursprung der Tibetischen Rezepturen.
Die Herstellung von Pflanzenformeln der Tibetischen Medizin nach modernen Qualitätsstandards ist komplex, ressourcenintensiv und anspruchsvoll. Wie auch bei konventionellen Arzneimitteln kommt dem Qualitätssicherungssystem eine zentrale Rolle zu. Die Vielstofflichkeit und das synergistische Wirkkonzept von Vielpflanzenrezepturen macht insbesondere die Definition von Qualitätskriterien und Kontrollparametern schwierig.

Neben der analytischen Qualitätskontrolle von Ausgangsstoffen und Fertigprodukt sowie der Prozessvalidierung der Herstellungsschritte wird eine kontinuierlich hohe Qualität in erster Linie damit erreicht, dass mögliche Fehlerquellen und qualitätsrelevante Risiken antizipiert werden und ihr Auftreten durch geeignete Maßnahmen verhindert wird.

Hierbei ist ein gutes Beziehungsnetz zu Produzenten und Lieferanten essenziell für die Qualität und die Qualitätssicherung der Ausgangsstoffe. Die Tatsache, dass die Firma Padma AG seit Jahrzehnten Rezepturen der Tibetischen Medizin nach modernen Qualitätsrichtlinien in der Schweiz herstellt, zeigt, dass es möglich ist, diese wertvollen Formeln des Tibetischen Arzneimittelschatzes auch für Menschen im Westen in gleichbleibender, hoher Qualität zur Verfügung zu stellen.

\section{Disclosure Statement}

A.S. und E.G. sind Mitarbeiter der Padma AG, Hinwil, Schweiz.

\section{Literatur}

-1 Schwabl H, Geistlich S, McHugh E: Tibetische Arzneimittel in Europa: Historische, praktische und regulatorische Aspekte. Forsch Komplementmed 2006;13(suppl 1):1-6.

2 Saller R: Tibetische Heilmittel bei chronischen Erkrankungen. Forsch Komplementmed 2006;13 (suppl 1):VII-VIII.

3 Csermely P, Ágoston V, Pongor S: The efficiency of multi-target drugs: the network approach might help drug design. Trends Pharmacol Sci 2005;26: 178-182.

4 European Medicines Agency: Note for guidance on good manufacturing practice for active pharmaceutical ingredients. 01/11/2000, Reference number CPMP/ICH/4106/00, www.ema.europa.eu.
5 Schwabl H, Vennos C, Saller R: Tibetische Rezepturen als pleitrope Signaturen - Einsatz von Netzwerk-Arzneien bei Multimorbidität. Forsch Komplementmed 2013;20(suppl 2):35-40.

6 European Pharmacopoeia / Pharmacopée européenne. Strasbourg, Council of Europe, 2004. www.edqm.eu/en/background-50.html.

7 Eloff JN, Ntloedibe DT, van Brummelen R: A simplified but effective method for the quality control of medicinal plants by planar chromatography. Afr J Tradit Complement Altern Med 2011;8(suppl 5):1-12.
8 Committee on Herbal Medicinal Products (HMPC): Concept paper on non-pharmacopoeial reference standards for herbal substances, herbal preparations and herbal medicinal products / traditional herbal medicinal products (Draft). Doc. Ref. EMEA/HMPC/312890/2012. www.ema.europa.eu.

9 Committee on Herbal Medicinal Products (HMPC): Guideline on good agricultural and collection practice (GACP) for starting materials of herbal origin. Doc. Ref. EMEA/HMPC/246816/2005. www.ema.europa.eu. 